\title{
ÍNDICE DE SELEÇÃO BIOECONÔMICO PARA FÊMEAS DE CORTE DA RAÇA NELORE
}

\author{
BIOECONOMIC SELECTION INDEX FOR NELLORE BEEF CATTLE FEMALES
}

Tanaka, A.L.R. ${ }^{1 \mathrm{~A}}$, Neves, H.H.R. ${ }^{1 \mathrm{~B}}$, Oliveira, J.A. ${ }^{2}$, Carvalheiro, R. ${ }^{3}$ e Queiroz, S.A. ${ }^{1 \mathrm{C}}$

1'Departamento de Zootecnia. Faculdade de Ciências Agrárias e Veterinárias, UNESP. Jaboticabal, SP. Brasil. Aailaloise@gmail.com; Bharoldozoo@hotmail.com; 'saquei@fcav.unesp.br

2Departamento de Ciências Exatas. Faculdade de Ciências Agrárias e Veterinárias, UNESP. Jaboticabal, SP. Brasil. jaoliv@fcav.unesp.br

${ }^{3}$ Gensys Consultores Associados S/S Ltda. Jaboticabal, SP. Brasil. carvalheiro@gensys.com.br

\section{Palavras chave adicionaIS}

Custo de mantença. Habilidade de permanência. Precocidade sexual. Produtividade materna.

\section{RESUMO}

A eficiência reprodutiva da fêmea Nelore foi descrita com base na precocidade sexual, permanência produtiva no rebanho (NP), produtividade materna (PM) e custo de mantença estimado (CM). A combinação dessas características deu origem ao índice bioeconômico retorno maternal (RMat), que estima o retorno em quilos de peso vivo produzidos por uma vaca em um ano. Em adição, incluiu-se a composição do peso produzido, adicionando à PM os escores de conformação, precocidade e musculatura a desmama, compondo o biótipo do bezerro. Foram consideradas precoces as fêmeas cuja idade ao primeiro parto foi inferior a 30 meses. A NP foi expressa pelo número de partos até 53 meses de idade. O CM foi calculado em função do consumo estimado de matéria seca da vaca. O RMat médio estimado foi $62,02 \pm 24,12 \mathrm{~kg} / \mathrm{vaca} / \mathrm{ano}$. As estimativas da variância genética aditiva e residual do RMat, usando a metodologia da máxima verossimilhança restrita, sob um modelo animal unicaracterística, foram 195,35 e 242,96 , respectivamente. A herdabilidade estimada para Rmat foi $0,45 \pm 0,02$, indicando que o índice é herdável e pode ser aplicado na seleção para eficiência reprodutiva. A NP foi o componente de principal variação do RMat. Touros selecionados com base no RMat apresentaram filhas mais eficientes.

\section{SUMMARY}

The reproductive efficiency of Nellore females

\section{AdDitiOnAL KEYWORDS}

Cost of maintenance. Maternal productivity. Stayability. Sexual precocity.

was described on the basis of sexual precocity staying productive in the herd (NP), maternal productivity (PM) and estimated cost for maintenance $(\mathrm{CM})$. The combination of these traits resulted in the bioeconomic maternal revenue index (RMat) ensures reproductive efficiency. The index estimates the return in kilograms of live weight produced per cow per year. In addition, the composition of calf weight produced was considered, adding to the PM the information on the scores of conformation, muscling and early finishing at weaning, in order to input the calf biotype. The females considered precocious had their age at first calving before 30 months. The NP was expressed by the number of calving till 53 months of age. The CM was calculated according to the estimated feed intake of dry matter. The estimated average RMat was $62.02 \pm 24.12 \mathrm{~kg} /$ cow/year. Estimates of additive genetic and residual variances for RMat, using restricted maximum likelihood under a single trait animal model was equal to 195.35 and 242.96 , respectively. The heritability estimated was $0.45 \pm 0.02$, indicating that RMat is inheritable and can be applied in the breeding program to improve reproductive efficiency. The NP was the main component of variation for RMat. Sires selected based on RMat tended to have more efficient daughters.

\section{INTRODUÇÃO}

Considerando que o sucesso da pecuária 
de corte está relacionado à produção de carne, o desafio dos criadores se resume, basicamente, em oferecer um produto de qualidade e em quantidade suficiente para manter a escala de produção e equilíbrio da balança comercial, tirando maior proveito das oportunidades que o mercado oferece. Conscientes que a produção de carne é função da produção de bezerros pelas fêmeas, os criadores e a comunidade científica vêm trabalhando no conceito de produtividade, na busca por vacas eficientes.

Segundo Perotto (2008), a eficiência para a produção de carne bovina pode ser definida em termos biológicos (peso vivo produzido/alimento consumido) ou em termos econômicos (receitas/despesas). Os aspectos produtivos de uma fêmea eficiente referem-se à capacidade materna que possui, mensurada pelo desempenho de sua progênie expressa em quilos de bezerros desmamados por hectare por ano, visto que em sistemas de cria, os bezerros desmamados constituem a principal fonte de renda para o produtor (Valle et al., 1998). Ortiz Peña et al. (2008) consideraram também, como forma de identificar vacas eficientes, o uso de escores visuais de carcaça no desempenho esperado na progênie, associados ao maior ganho de peso.

A eficiência reprodutiva é um dos componentes mais importantes que afetam a produção (Mercadante et al., 1996; Donoghue, 2006), sendo considerada a fertilidade, a qual determina a quantidade de animais produzidos, como um dos fatores responsáveis pela lucratividade da atividade (Silva et al., 2003). Deste modo, uma vez que as matrizes representam a categoria animal que consome a maior parte dos recursos alimentares disponíveis ao rebanho, o custo de mantença destas, é um dos principais fatores de influência nos custos de produção. Ortiz Peña et al. (2008) consideraram o custo de mantença como uma função do peso adulto e do consumo estimado de matéria seca e incluíram este componente em um índice de eficiência produtiva, visando o aumento de produtividade sem incremento de custos e sem uma possível resposta correlacionada negativa na fertilidade das vacas em decorrência do aumento do peso adulto.

Em um rebanho sob seleção, é fundamental identificar fêmeas eficientes, reproduzi-las, e também descartar as inferiores. Segundo Fries (2003), a eliminação de vacas falhadas/improdutivas permitiria aumentar o nível alimentar das novilhas de um a dois anos, favorecendo a reprodução e, consequentemente, a produção de bezerros, promovendo aumento considerável na taxa de desfrute, ou seja, na lucratividade. Portanto, os trabalhos de seleção devem caracterizar uma fêmea eficiente para a produção de carne, como aquela que inicia sua vida reprodutiva jovem, possui assiduidade reprodutiva e sua progênie apresenta desempenhos de carcaça diferenciados quanto à produção de carne, considerando os custos gerados nesse processo.

A eficiência reprodutiva de fêmeas pode ser tratada então, como um objetivo de seleção, definido por Ponzoni e Newman (1989), como a combinação de características biológicas e economicamente importantes dentro de um sistema de produção. Esse objetivo pode ser expresso em um índice de seleção, ou seja, um valor único que prediz o mérito genético global de um indivíduo para uma combinação ponderada de características (Bourdon, 1997). É na análise de quais características devem ser utilizadas para selecionar fêmeas eficientes, que pesquisadores trabalham atualmente.

Teixeira et al. (2002) compararam aspectos bioeconômicos entre três sistemas de produção em bovinos da raça Nelore, ou seja, novilhas cobertas aos 15, 18 e 27 meses de idade, considerando um rebanho a pasto e sob variações no nível de fertilidade. Foi verificado que sob essas circunstâncias é possível incrementar a receita bruta da atividade mediante a antecipação do primeiro parto, intensificando o processo produtivo. Também foi observado que a 
partir de determinados níveis, os aumentos de fertilidade não acarretaram em grandes alterações nos níveis de desfrute. Abreu et al. (2003) em uma análise bioeconômica da introdução de período de monta (PM) em sistemas de produção de rebanhos de cria na região do Brasil central, verificaram que a implementação de $\mathrm{PM}$ proporcionou melhoria substancial na economicidade e na eficiência biológica do sistema, sendo o aumento na taxa de natalidade o efeito de maior impacto positivo na atividade. Brumatti et al. (2011), desenvolvendo um modelo bioeconômico para determinação de ponderadores econômicos utilizados em índices de seleção em programas de melhoramento genético de bovinos de corte, verificaram que as características reprodutivas, habilidade de permanência e probabilidade de prenhez aos 14 meses de idade, foram mais importantes economicamente do que as características de crescimento, peso a desmama e ao sobreano e ganho de peso pós-desmama.

Este trabalho teve como objetivo determinar um índice bioeconômico (RMat) que expresse a eficiência reprodutiva, mensurada pelo retorno dado por uma vaca Nelore, em quilos de peso vivo produzido por ano, descontado o custo estimado de mantença.

\section{MATERIALE MÉTODOS}

\section{DESCRICOAODOBANCO DEDADOS}

O presente trabalho utilizou informações de animais da raça Nelore, pertencentes ao banco de dados do programa de melhoramento genético de bovinos de corte PAINT - da CRV Lagoa. Por ser um programa comercial sua base de dados pode ser caracterizada como aberta, de modo que a cada ano, novas fazendas ingressam no programa, contribuindo para o aumento da diversidade genética existente entre os rebanhos participantes do programa.

O manejo alimentar adotado nestas fazendas consistiu-se basicamente, de pasto, sal mineral e água para consumo ad libitum. Em geral, não foi realizada suplementação energética para qualquer categoria de fêmeas. No entanto, em algumas fazendas onde não havia uniformidade de qualidade e disponibilidade de forragens, foram separados os melhores pastos para as fêmeas em reprodução.

Quanto ao manejo reprodutivo, parte dos rebanhos adotou duas estações reprodutivas: uma antecipada que, em geral, ocorre de março a junho, utilizada para identificar a precocidade sexual de novilhas e, a estação tradicional, de outubro a fevereiro. Os rebanhos restantes adotaram somente a estação reprodutiva tradicional. A grande maioria das fêmeas foi inseminada e, caso não emprenhassem, foram expostas a reprodutores em sistema de repasse. A monta natural, quando se expõe um grupo de fêmeas a um único reprodutor, é denominada controlada e, nesse caso, a paternidade é conhecida. Porém, em sua maioria, os repasses foram realizados com mais de um touro por grupo de fêmeas, manejo denominado repasse com touro múltiplo, em que a paternidade somente poderia ser conhecida por meio de exames de DNA.

As novilhas que emprenharam na estação antecipada foram favorecidas, uma vez que tiveram maior período para se recuperarem e conceberem no início da estação de monta tradicional quando primíparas, aumentando as condições para manterem uma vida produtiva longa dentro do rebanho. A maioria dos rebanhos participantes do programa expôs as primíparas à monta natural controlada ou com grupos de reprodutores, aumentando assim, a taxa de prenhez dessa categoria. Essas práticas não geraram investimentos diretos aos criadores, somente uma mudança nas atividades da equipe de campo para implantação dos grupos de fêmeas e touros que participaram desta estação reprodutiva. De maneira geral, após 60 dias a partir do término da estação reprodutiva, as fêmeas passaram por diagnóstico de gestação mediante palpação retal ou ultrassonografia. 
Informações como identificação da mãe, data do parto, escore de peso ao nascer e grupo de manejo foram coletadas por ocasião do nascimento dos bezerros. Ao redor de 6-8 meses, estes foram desmamados e apartados das mães que retornaram aos pastos. Após jejum total, de aproximadamente, 12 horas, os bezerros foram submetidos ao manejo sanitário (vacinação e desverminação), pesados e avaliados quanto à conformação $(\mathrm{C})$, precocidade $(\mathrm{P})$ e musculatura $(\mathrm{M})$, por escores variando de 1 a 5 , atribuídos de forma relativa aos demais animais pertencentes ao mesmo grupo de manejo. Em C, avalia-se o quanto o animal produziria de carne se fosse abatido naquele momento. Essa característica é influenciada pelo tamanho do animal, principalmente o comprimento e profundidade de costelas. $\mathrm{Na}$ avaliação de $\mathrm{P}$, tenta-se predizer a capacidade do animal chegar a um grau de acabamento mínimo de carcaça, com peso vivo não elevado. $\mathrm{O}$ acabamento refere-se ao desenvolvimento do tecido adiposo, em termos de quantidade e distribuição nas regiões corporais do animal. Por $\mathbf{M}$, avaliase o desenvolvimento da massa muscular como um todo, observada em pontos como antebraço, paleta, lombo, garupa e, principalmente, no traseiro (Queiroz et al., 2009).

\section{EXPLORAÇÃO DE DADOS E ANÁLISES PRE- LIMINARES}

A edição e exploração de dados foram realizadas no software SAS. Partiu-se de um arquivo formado por todos os bancos de dados dos rebanhos participantes do programa. Os dados foram submetidos às averiguações costumeiras e as informações incoerentes ou biologicamente discrepantes foram eliminadas.

Para avaliar a permanência produtiva, foram eliminados do banco de dados, fêmeas nascidas após março de 2006, restando apenas as com idade suficiente para terem sido desafiadas pelo menos duas vezes. Eliminaram-se novilhas cuja idade ao primeiro parto foi inferior a 21 ou superior a 40 meses, e aquelas cujas idades à segunda e à terceira concepção foram menores que 31 e 41 meses, respectivamente. Apenas os partos até os 53 meses de idade foram considerados.

Foram calculados os intervalos de parto e quando esses foram inferiores a 10 meses, foram desconsiderados. Assumiu-se que as fêmeas com nascimento controlado no programa que não apareceram como vacas no arquivo de dados foram descartadas/ abatidas com 30 meses de idade, e não produziram nenhum bezerro.

O grupo de contemporâneos (GC) foi formado pela concatenação das variáveis: rebanho, ano, estação de nascimento e grupos de manejo ao desmame e ao sobreano. A estação de nascimento foi definida por duas classes, sendo seca atribuída aos animais que nasceram entre os meses de abril e setembro e águas, para aqueles nascidos entre outubro e março. Realizou-se a verificação da conectabilidade de dados entre os GCs, bem como a existência de laços genéticos entre eles. Para isso, foram excluídos GCs com menos de 5 fêmeas, touros com menos de 5 filhas e com filhas de somente um ou dois touros, restando 7328 GCs.

Desta forma, o arquivo de dados para a análise do índice retorno maternal contou com 60361 observações de fêmeas Nelore, nascidas no programa PAINT entre os anos de 1992 a 2006, filhas de 548 touros de sêmen ou monta controlada.

\section{ÍNDICE BIOECONÔMICO-RMAT}

$\mathrm{O}$ índice bioeconômico proposto visa identificar a eficiência reprodutiva em fêmeas da raça Nelore, definida pela precocidade sexual, permanência produtiva no rebanho e pela capacidade de desmamar bezerros com elevado desempenho para produção de carne. Alia-se ainda ganho de peso a escores visuais de carcaça (conformação, precocidade e musculatura), avaliados a desmama, considerando-se o custo de mantença dessas fêmeas.

A combinação destas características resulta em um valor único, o retorno mater- 


\section{ÍNDICE DE SELEÇÃO BIOECONÔMICO PARA FÊMEAS DE CORTE DA RAÇA NELORE}

nal (RMat), que é uma estimativa do retorno em quilos de peso vivo produzido por vaca ao ano, descontado o custo estimado de mantença.

Para o cálculo do retorno maternal (RMat) proposto por Carvalheiro et al. (2005 a, b), as fêmeas foram avaliadas sob os seguintes aspectos:

Precocidade sexual: Para este componente, adotou-se um ponderador econômico calculado como função da idade da vaca ao parto. As fêmeas com idades ao primeiro parto inferiores a 30 meses foram consideradas precoces, as com idades iguais ou superiores a 30 meses foram classificadas no grupo normal e aquelas que não chegaram a se reproduzir foram denominadas falhadas. Foi acrescido um bônus de $1 \%$ por mês, expresso em precocidade, aos pesos preditos de produtos de fêmeas precoces em relação às fêmeas consideradas no grupo normal, de acordo com Ortiz Peña et al. (2008).

Habilidade de permanência produtiva no rebanho: Foram considerados os números de partos das fêmeas com até 53 meses de idade, de forma a contemplar o período crítico de reconcepção de primíparas e antecipar a avaliação genética de touros baseada em mensurações da característica em suas filhas.

Produtividade materna: Foi obtida pelos desempenhos preditos da progênie calculados como função das diferenças esperadas na progênie (DEPs) para ganho médio de peso no período pré-desmama, conformação, precocidade e musculatura a desmama. Foram utilizadas as DEPs das vacas em vez dos desempenhos dos bezerros visando eliminar os possíveis efeitos ambientais, a influência do mérito genético dos touros acasalados com que essas fêmeas e a influência da segregação mendeliana do indivíduo. Admitiu-se produtividade materna igual a zero para as fêmeas que não conceberam, sendo, no entanto, utilizados, nesse caso, os respectivos pesos estimados de descarte, pois os mesmos geraram renda, da qual foram descontados $10 \%$, uma vez que os demais cálculos foram baseados em arroba de boi gordo.

Retorno maternal (kg/vaca/ano): O índice bioeconômico retorno maternal (RMat) foi estimado por meio da expressão:

$R M a t=\frac{\left[N P x\left(Y_{\text {bezerro }} x \text { Pond } \$\right)+Y_{\text {vaca }}\right] \quad C M}{(\text { ivp }+T C)} \times 12$ em que:

RMat= Retorno anual em quilos de peso vivo produzido por vaca, considerando a composição em peso e o custo de mantença;

$\mathrm{NP}=$ Número de produtos da vaca até os 53 meses;

$Y_{\text {bezerro }}=$ Peso predito do bezerro produzido no parto i ( $i=1$ a NP). Para a estimação desse peso, considerou-se um índice de seleção combinando as DEPs padronizadas da vaca para ganho médio diário do nascimento a desmama (60\%), conformação (8\%), precocidade (16\%) e musculatura (16\%). Em caso de fêmeas precoces, o $Y_{\text {bezerro }}$ foi acrescido de um bônus de $1 \%$ por mês, expresso em precocidade.

Pond $\$=$ Ponderador econômico que considerou dois componentes: o valor de oportunidade do bezerro produzido, que neste trabalho foi constante e igual a uma arroba $(30 \mathrm{~kg})$ e um custo operacional relacionado ao manejo reprodutivo, expresso em quilos. Este custo operacional está relacionado ao custo de submeter uma fêmea à estação reprodutiva multiplicado pelo número de estações que participou. Os custos com a estação reprodutiva envolveram despesas com sêmen, material utilizado na inseminação artificial (aplicador, luvas, etc.), bem como mão-deobra do inseminador e auxiliar e uso de touro, em caso de monta controlada. Assumiu-se um custo operacional de $25 \mathrm{~kg}$.

$\mathrm{Y}_{\text {vaca }}=$ peso predito da vaca ao descarte;

$\mathrm{CM}=$ Custo de mantença da vaca calculado como uma função de seu peso adulto (PV) e do consumo estimado de matéria seca (ConsMS). O ConsMS diário utilizado foi equivalente a $9 \%$ do peso metabólico $\left(\mathrm{PV}^{0,75}\right)$, pressupondo-se custo do $\mathrm{kg}$ de matéria seca de $0,02 \mathrm{~kg}$ de peso vivo. Os PVs foram obtidos por simulações que consideraram uma distribuição normal com média de 420 kg e desvio-padrão de $42 \mathrm{~kg}$, pressupondo-se correlação de $0,70 \mathrm{com} o$ 
peso das fêmeas obtidos pela padronização (desvio-padrão= 1,0 ) de suas DEPs para ganho de peso.

ivp= idade da vaca ao parto; e

$\mathrm{TC}=$ constante representando o tempo para o último produto nascido desmamar e ser comercializado, a partir da data do parto. Foi utilizado um período igual a 12 meses.

Posteriormente, as fêmeas foram classificadas para eficiência reprodutiva de acordo com o desempenho para precocidade sexual, permanência produtiva e produtividade materna, totalizando 10 classes, cuja nomenclatura foi definida pela combinação das siglas que representaram as classes para precocidade sexual + permanência produtiva + produtividade materna . Cada componente foi descrito como:

- Precocidade sexual: $\mathrm{f}=$ falhada $(\mathrm{NP}=$ $0) ; \mathrm{p}=$ precoce $(\mathrm{ivp}<30$ meses de idade $)$;

- $\mathrm{n}=$ normal (ivp $\geq 30$ meses de idade);

- Permanência produtiva: representada pelo número de partos i (i=0 a 2);

- Produtividade materna: foi utilizado o sinal (+) para indicar superioridade $\left(\mathrm{Y}_{\mathrm{be}}\right.$ $>169 \mathrm{~kg}$ ) e o sinal (-) para inferioridade $\left(\mathrm{Y}_{\text {bezerro }} \leq 169 \mathrm{~kg}\right.$ ) em desempenho da progênie.

$\mathrm{O}$ arquivo de dados do RMat foi analisado pelo programa computacional Multiple Trait Derivative Free Restricted Maximum Likelihood (MTDFREML) (Boldman et al., 2002) e assim, as estimativas dos componentes de variância e da herdabilidade do índice foram obtidas pelo método da máxima verossimilhança restrita, por meio de um modelo animal uni-característica, com equações de modelos mistos, cuja matriz de parentesco incluiu 108002 animais. O modelo empregado nas análises considerou o GC como efeito fixo e o efeito genético aditivo como aleatório e pode ser representado por:

$$
\mathrm{Y}=\mathrm{X} \beta+\mathrm{Za}+\mathrm{e}
$$

em que:

$Y=$ vetor de observações:

$X=$ matriz de incidência associando observações ao efeito fixo de GC;

$\beta=$ vetor de efeito fixo de grupo de contemporâneos;
Z= matriz de incidência associando observações ao efeito genético aditivo direto;

$\mathrm{a}=$ vetor de efeito genético aditivo direto do animal; $\mathrm{e}=$ vetor de resíduos aleatórios.

Assumiram-se as seguintes pressuposições:

$$
\begin{aligned}
& \mathrm{E}(\mathrm{a})=0 ; \mathrm{E}(\mathrm{e})=0 \\
& \operatorname{Var}(\mathrm{a})=\mathrm{A} \cdot \sigma_{\mathrm{a}}^{2} ; \operatorname{Var}(\mathrm{e})=\mathrm{I}_{\mathrm{n}} \cdot \sigma_{\mathrm{e}}^{2}
\end{aligned}
$$

em que:

$E(a)=$ esperança do efeito genético aditivo direto de animal;

$E(e)=$ esperança do efeito residual;

$a=$ efeito genético aditivo direto de animal;

$\mathrm{A}=$ numerador da matriz de parentesco;

$\sigma_{\mathrm{a}}^{2}=$ variância genética aditiva direta;

$\mathrm{e}=$ efeito residual;

$\mathrm{I}_{\mathrm{n}}=$ matriz identidade de ordem igual ao número de observações;

$\sigma_{e}^{2}=$ variância residual.

Considerou-se que o critério de convergência foi alcançado quando a variância dos valores da função (-2 $\log \mathrm{L})$ do algoritmo simplex foi igual ou menor que $10^{-12}$. Como há possibilidade de se obterem valores máximos locais, ao invés do máximo global, as análises foram repetidas várias vezes no sentido de assegurar a convergência no máximo global da função de verossimilhança.

As diferenças esperadas na progênie para RMat foram calculadas e esses valores ordenados de forma decrescente em 10 níveis, denominados decis ou decas. Os desempenhos das progênies de touros pertencentes a diferentes decas foram comparados.

\section{RESULTADOSEDISCUSSÃO}

\section{ESTATÍSTICASDESCRITIVAS}

O valor médio da idade da vaca ao primeiro parto (IVP1) encontrado neste trabalho, 35,08 meses (tabela I), foi semelhante ao valor estimado por Forni e Albuquerque (2005) também em estudo com a raça Nelore (34,88 meses). Outros autores 


\section{ÍNDICE DE SELEÇÃO BIOECONÔMICO PARA FÊMEAS DE CORTE DA RAÇA NELORE}

como Garnero et al. (1999) e Boligon et al. (2007) estimaram IVP1 de 36 meses. Valores maiores foram relatados por Bergmann et al. (1998) e Silveira et al. (2004), 38 e 41 meses, respectivamente, para fêmeas da mesma raça. Neste último estudo, o valor elevado de IVP1 foi atribuído ao sistema extensivo de criação e as restrições alimentares em período de escassez de pastagem. Considerando que o número máximo de parições por vaca foi igual a dois e a idade máxima das fêmeas igual a 53 meses, o intervalo médio entre a primeira e a segunda parição (IEP) obtido neste estudo (14,30 meses) pode ser considerado bom para uma vaca eficiente. Silveira et al. (2004) obtiveram IEP de 15,24 meses, sem a aplicação de restrições para a formação do banco de dados.

$\mathrm{O}$ retorno maternal (RMat) calculado para 60361 fêmeas apresentou valor médio de $62,02 \pm 24,12 \mathrm{~kg} / \mathrm{vaca} / \mathrm{ano}$, sendo que estatísticas descritivas dessa variável foram estimadas dentro das classes de precocidade sexual (tabela II). Observou-se que, apenas, 1,97\% das fêmeas analisadas foram consideradas precoces, sendo em média $63,13 \mathrm{~kg}$ mais eficientes em relação às fêmeas que não chegaram a se reproduzir (falha-

Tabela I. Estatísticas descritivas das variáveis IVP1, IVP2 e IEP, expressas em meses, utilizadas nos cálculos dos componentes do RMat de fêmeas da raça Nelore. (Descriptive statistics of variables IVP1, IVP2 and IEP, expressed in months, used in the calculations for Nellore females RMat components).

\begin{tabular}{lccccc}
\hline & No obs. Média & DP & Mín. & Máx. \\
\hline IVP1* $^{*} 20761$ & 35,08 & 2,73 & 21,00 & 40,00 \\
IVP2* $^{*}$ & 7193 & 48,41 & 2,28 & 35,00 & 53,00 \\
IEP & 7193 & 14,30 & 2,49 & 10,00 & 27,00
\end{tabular}

*IVP1 e IVP2: idade da vaca ao primeiro e ao segundo parto, respectivamente.

IEP: intervalo entre a primeira e a segunda parição. № obs.= número de observações; DP: Desviopadrão.
Tabela II. Estatísticas descritivas do retorno maternal de vacas Nelore classificadas, de acordo com a precocidade sexual. (Descriptive statistics for maternal revenue of Nellore cows classified according to sexual precocity).

\begin{tabular}{lccccc}
\hline & \multicolumn{5}{c}{ Retorno maternal* } \\
& № obs. Média & DP & Mín. & Max. \\
\hline falhada & 39600 & 48,08 & 9,54 & 6,80 & 103,68 \\
normal & 19573 & 87,22 & 20,05 & 24,69 & 185,14 \\
precoce & 1188 & 111,21 & 23,54 & 51,76 & 182,97 \\
\hline
\end{tabular}

*kg peso vivo/vaca/ano.

№ obs.= número de observações; DP: Desviopadrão.

das). Porém, fêmeas classificadas no grupo normal, ou seja, com idade ao primeiro parto igual ou maior que 30 meses apresentaram, em relação às precoces, Rmat $23,99 \mathrm{~kg}$ menor e superioridade, em relação às falhadas, de $81 \%$ (tabela II).

Avaliando a variação dos valores de RMat, quanto à permanência produtiva no rebanho até 53 meses de idade (tabela III), pode-se observar maior retorno maternal

Tabela III. Estatísticas descritivas do retorno maternal de vacas Nelore, de acordo com a permanência produtiva no rebanho até 53 meses de idade, expressa pelo número de partos. (Descriptive statistics of the maternal revenue of Nellore cows, according to stayability in the herd up to 53 months of age, expressed as the number of calving).

\begin{tabular}{cccccc}
\hline \multicolumn{5}{c}{ Retorno maternal* } \\
& No obs. & Média & DP & Mín. & Max. \\
\hline 0 & 39600 & 48,08 & 9,54 & 6,80 & 103,68 \\
1 & 13568 & 78,57 & 14,71 & 24,69 & 167,00 \\
2 & 7193 & 107,49 & 17,89 & 45,11 & 185,14
\end{tabular}

${ }^{*} \mathrm{~kg}$ peso vivo/vaca/ano.

№ obs.= número de observações; DP: desviopadrão. 
médio resultante de duas parições, sendo este $37 \%$ superior em relação a vacas com apenas um parto e $124 \%$ em relação as fêmeas que não se reproduziram, indicando que a produção de carne gerada por uma unidade a mais de bezerro é um dos fatores que mais contribuem para o aumento do RMat das vacas.

Observando-se os RMats de acordo com as classes de eficiência reprodutiva (tabela IV), é possível verificar as variações resultantes da interação entre os componentes precocidade sexual, permanência produtiva e produtividade materna. De modo condizente com as observações biológicas, os melhores resultados de RMat foram obtidos por fêmeas com maior número de partos, precoces e que produziram bezerros com mérito superior.

As fêmeas que não chegaram a conceber (tabela IV) geraram receita proporcional ao desempenho de sua carcaça (0f+e 0f-). Ortiz Peña et al. (2008) estimaram RMat médio próximo ao desse trabalho para fêmeas of-,
$43 \mathrm{~kg}$. O desempenho da progênie contribui para elevar os valores de RMat, quando se compara produtividade materna superior e inferior para a mesma classe de precocidade e permanência.

Vacas precoces com dois partos e progênie com desempenho em ganho de peso e biótipo da carcaça para produção de carne acima da média $(2 \mathrm{p}+)$ foram aquelas com maior RMat médio estimado, 130,93 kg/ vaca/ano. Estas foram $32 \%$ superiores a fêmeas precoces, com progênie também superior, mas que pariram somente um bezerro em sua vida produtiva $(1 \mathrm{p}+)$, conforme pode ser observado na tabela IV. Para a classe 2p+, Ortiz Peña et al. (2008) encontraram valor inferior (121 kg/vaca/ano) ao deste trabalho. A comparação entre as classes de fêmeas precoces $1 \mathrm{p}+(98,93 \mathrm{~kg} /$ vaca/ano) e normais $2 \mathrm{n}-(95,08 \mathrm{~kg} / \mathrm{vaca} /$ ano) permite verificar a importância do número de partos no Rmat, confirmando os resultados obtidos por Formigoni et al. (2005). Estes pesquisadores concluíram que

Tabela IV. Estatísticas descritivas do retorno maternal de vacas Nelore, de acordo com classes de eficiência reprodutiva (ER), permanência reprodutiva $(P R)$, precocidade sexual $(P S)$ e produtividade materna (PMat). (Descriptive statistics of maternal return of Nellore cows, according to classes of reproductive efficiency (ER), stayability (PR), sexual precocity (PS) and maternal productivity (PMat)).

\begin{tabular}{lcllccccc}
\hline & & & & \multicolumn{5}{c}{ Retorno maternal $(\mathrm{kg}$ peso vivo/vaca/ano) } \\
ER $^{1}$ & PR $^{2}$ & PS & PMat $^{3}$ & No obs. & Média & DP & Mín. & Máx. \\
\hline Of- & 0 & falhada & inferior & 27792 & 44,25 & 6,94 & 6,80 & 61,48 \\
Of+ & 0 & falhada & superior & 11808 & 57,12 & 8,68 & 35,23 & 103,68 \\
$1 n-$ & 1 & normal & inferior & 7997 & 70,67 & 9,52 & 24,69 & 105,01 \\
$1 n+$ & 1 & normal & superior & 5137 & 89,85 & 13,22 & 57,50 & 167,00 \\
$1 \mathrm{p}-$ & 1 & precoce & inferior & 179 & 79,14 & 11,17 & 51,76 & 109,69 \\
$1 \mathrm{p}+$ & 1 & precoce & superior & 255 & 98,93 & 11,81 & 77,83 & 141,90 \\
$2 n-$ & 2 & normal & inferior & 3465 & 95,08 & 10,58 & 45,11 & 130,36 \\
$2 n+$ & 2 & precoce & superior & 2974 & 118,02 & 14,17 & 84,61 & 185,14 \\
$2 p-$ & 2 & precoce & inferior & 218 & 103,44 & 12,30 & 69,65 & 141,81 \\
$2 \mathrm{p}+$ & 2 & precoce & superior & 536 & 130,93 & 15,19 & 93,50 & 182,97 \\
\hline
\end{tabular}

№ obs.= número de observações; DP: desvio-padrão.

${ }^{1} \mathrm{f}=$ falhada; $\mathrm{n}=$ normal; $\mathrm{p}=$ precoce; $+=$ superior; $-=$ inferior; ${ }^{2}$ Número de partos até os 53 meses de idade; ${ }^{3}$ Desempenho da progênie estimado com base no desempenho da mãe. 
a habilidade de permanência em vacas Nelore apresentou maior retorno econômico que a precocidade sexual e verificaram ainda que o valor econômico da habilidade de permanência variou em função do custo de compra da novilha de reposição, ou seja, quanto maior for o investimento na reposição desta categoria, maior será a importância econômica da habilidade de permanência. Corroborando com essa conclusão, Ortiz Peña et al. (2008) afirmaram que não basta a fêmea ser precoce e produzir bezerros com desempenho superior, se não apresentar permanência produtiva, bem como vacas precoces com produtividade materna inferior apresentam retorno menor do que vacas não precoces com progênie de mérito superior. No presente estudo, essa constatação também foi confirmada pela comparação das fêmeas das classes $2 \mathrm{n}+\mathrm{e}$ $2 \mathrm{p}-$, com Rmat iguais a $118,02 \mathrm{~kg} / \mathrm{vaca} / \mathrm{ano}$ e $103,44 \mathrm{~kg} / \mathrm{vaca} / \mathrm{ano}$, respectivamente (tabela IV).

Os custos anuais estimados de mantença praticamente não diferiram entre as classes (tabela V) de eficiência reprodutiva, indicando a possibilidade de aumento da eficiência produtiva e reprodutiva sem aumento do custo.

\section{ESTIMATIVAS DOS COMPONENTES DE VARIÂNCIA E HERDABILIDADE}

Para o índice bioeconômico retorno maternal (RMat), as estimativas das variâncias genética aditiva, residual e fenotípica foram iguais a 195,35; 242,96 e 438,30, respectivamente. Cabe ressaltar que as estimativas dos componentes de variância obtidas para um índice refletem a variação de diversas características em conjunto, sendo que, em algumas situações, podem indicar uma variação maior do que seria esperado para algumas das características individualmente. Azevêdo et al. (2005) estudaram um índice de produtividade de fêmeas bovinas Nelore, denominado produtividade acumulada ( $\mathrm{kg}$ de bezerros desmamados por ano), que incluiu as carac- terísticas: média de peso dos bezerros ao desmame, número total de bezerros produzidos pela vaca, uma constante igual a 365 dias visando expressar a fertilidade em base anual, idade da vaca ao último parto e uma constante igual a 550 dias, utilizada com base na expectativa de o primeiro parto ocorrer ao redor de 30 meses de idade. Os autores estimaram, para esse índice, componentes de variância aditiva e de ambiente iguais a 95,16 e 748,01, respectivamente, argumentando que fórmulas que visam a avaliar a produtividade total devem ser utilizadas com certa precaução, pois incorporam muitas características de diferentes variabilidades e importâncias econômicas. Nesses casos, a herdabilidade do índice tende a ser controlada pela característica mais variável e menos herdável incluída na equação (Notter, 1995).

O coeficiente de herdabilidade do RMat foi estimado em $0,45 \pm 0,02$, o qual indica que o índice é herdável e pode ser utilizado como

Tabela V. Estatísticas descritivas dos custos anuais estimados de acordo com a classe de eficiência reprodutiva da fêmea. (Descriptive statistics of the estimated annual costs according to the class of reproductive efficiency of the female).

\begin{tabular}{cccc}
\hline ER $^{1}$ & № obs. & Média & DP \\
\hline Of- & 27792 & 59,31 & 1,23 \\
Of+ & 11808 & 60,60 & 1,20 \\
$1 \mathrm{n}-$ & 7997 & 59,43 & 1,36 \\
$1 \mathrm{n}+$ & 5137 & 60,90 & 1,45 \\
$1 \mathrm{p}-$ & 179 & 58,94 & 1,60 \\
$1 \mathrm{p}+$ & 255 & 60,92 & 1,39 \\
$2 \mathrm{n}-$ & 3465 & 59,34 & 1,47 \\
$2 \mathrm{n}+$ & 2974 & 60,98 & 1,49 \\
$2 \mathrm{p}-$ & 218 & 58,64 & 1,49 \\
$2 \mathrm{p}+$ & 536 & 60,99 & 1,49 \\
\hline
\end{tabular}

ER: eficiência reprodutiva; № obs.= número de observações; DP: desvio-padrão.

${ }^{1} \mathrm{f}=$ falhada; $\mathrm{n}=$ normal; $\mathrm{p}=$ precoce $;+=$ superior; - = inferior 
ferramenta de seleção quando se objetiva reproduzir e fixar a característica eficiência reprodutiva em fêmeas. Para o índice produtividade acumulada, Azevêdo et al. (2005) estimaram coeficiente de herdabilidade igual a $0,11 \pm 0,06$.

É importante mencionar que a estimativa de herdabilidade do Rmat pode estar acima dos valores de herdabilidade das características isoladas que o compõem, como precocidade sexual e permanência produtiva no rebanho. Por outro lado, pode ter sido reflexo da ação gênica presente na produtividade materna, que foi avaliada por características de desempenho do bezerro que, em geral, possuem estimativas para esse parâmetro, de magnitudes moderadas a altas. O exposto sugere que a combinação dos fenótipos em uma única característica, ou seja, a composição de um índice fenotípico pode ser uma alternativa, quando a composição de índices de seleção baseados nas diferenças esperadas na progênie, não é viável.

Ao comparar o desempenho das filhas dos 548 touros avaliados para o RMat, podese observar que os touros deca 1 apresentaram $4,81 \%$ de filhas precoces, enquanto os touros deca 10, 1,26\%. Quanto à permanência produtiva, os touros deca 1 apresentaram 21,04\% das filhas com dois partos até os 53 meses, enquanto que, os touros deca 10 , apenas $9,44 \%$. Ortiz Peña et al. (2008) relataram valores de 38,8\% e $0,20 \%$, respectivamente, para essa mesma comparação.

Quanto ao RMat, touros deca 1 apresentaram filhas com valor médio de $81,50 \mathrm{~kg} /$ vaca/ano, $62 \%$ acima do desempenho médio da filhas dos touros deca 10. Ortiz Peña et al. (2008) mencionaram que touros deca 1

\section{BIBLIOGRAFIA}

Abreu, U.G.P., Cezar, I.M. e Torres, R.A. 2003. Análise bioeconômica da introdução de período de monta em sistemas de produção de rebanhos de cria na região do Brasil central. Rev Bras apresentaram RMat médio das filhas $(75,0$ $\mathrm{kg} / \mathrm{vaca} / \mathrm{ano}$ ) $71 \%$ superior em relação ao retorno estimado das filhas dos touros deca $10(43,9 \mathrm{~kg} / \mathrm{ano})$. Esses resultados sugerem que a seleção de touros pode ser baseada no índice RMat, o qual pode ainda, ser utilizado como ferramenta auxiliar no direcionamento de acasalamentos, bem como na seleção de fêmeas para práticas de biotecnologia da reprodução, como produção in vitro e uso de sêmen sexado.

\section{CONCLUSÕES}

A combinação da precocidade sexual, permanência produtiva no rebanho e produtividade materna no índice retorno maternal, levando-se em consideração o custo de mantença das vacas, foi eficaz na identificação de touros para eficiência reprodutiva.

A herdabilidade do retorno maternal indicou que este índice é herdável e pode ser aplicado para a seleção de touros cujas filhas tenderam a ser precoces, produziram até os 4 anos de idade maior número de bezerros com desempenho superior para a produção de carne, sem aumentar os custos de produção.

A habilidade de permanência produtiva no rebanho até os 53 meses foi o componente que resultou em maior variação do retorno maternal, quando houve aumento, em uma unidade, do número de partos da fêmea.

\section{AGRADECIMENTOS}

Os autores agradecem ao Programa PAINT da CRV Lagoa pela cessão dos dados para a realização dessa pesquisa e ao CNPq e à FAPESP pela concessão de bolsa de pesquisa.
Zootecn, 32: 1198-1206.

Azevêdo, D.M.M.R., Martins Filho, R., Lôbo, R.N.B., Lôbo, R.B., Moura, A.A.A.N., Pimenta Filho, E.C. e Malhado, C.H.M. 2005. Produtividade acumu- 
lada (PAC) das matrizes em rebanhos Nelore do norte e nordeste do Brasil. Rev Bras Zootecn, 34: 54-59.

Bergmann, J.A.G., Gressler, S.L., Pereira, C.S., Penna, V.M. e Pereira, J.C.C. 1998. Avaliação de fatores genéticos e de ambiente sobre algumas características reprodutivas de fêmeas da raça Nelore em regime de estação de monta restrita. Arq Bras Med Vet Zootecn, 50: 633-645.

Boldman, K.G., Kriese, L.A., Van Vleck, L.D. and Kachman, S.D. 2002. A manual for use for MTDFREML: a set of programs to obtain estimates of variances and covariances. Department of Agricultural. Agricultural Research Service. Lincoln. 129 pp.

Boligon, A.A., Rorato, P.R.N. e Albuquerque, L.G. 2007. Correlações genéticas entre medidas de perímetro escrotal e características produtivas e reprodutivas de fêmeas da raça Nelore. Rev Bras Zootecn, 36: 565-571.

Bourdon, R.M. 1997. Understanding animal breeding. Prentice Hall. New Jersey. 523 pp.

Brumatti, R.C., Ferraz, J.B.S., Eler, J.P. e Formigonni, I.B. 2011. Desenvolvimento de índice de seleção em gado de corte sob o enfoque de um modelo bioeconômico. Arch Zootec, 60: 205-213.

Carvalheiro, R., Severo, J.L.P., Queiroz, S.A. e Fries, L.A. 2005a. Protótipo de um critério de seleção visando aumentar a eficiência produtiva de vacas de corte: I- Índice proposto. Em: Reunião Anual da Sociedade Brasileira de Zootecnia, Anais... Sociedade Brasileira de Zootecnia. Goiânia, GO. 42.

Carvalheiro, R., Severo, J.L.P., Queiroz, S.A. e Fries, L.A. 2005b. Protótipo de um critério de seleção visando aumentar a eficiência produtiva de vacas de corte: II- Comparação de índices alternativos. Em: Reunião Anual da Sociedade Brasileira de Zootecnia, Anais... Sociedade Brasileira de Zootecnia. Goiânia, GO. 42.

Donoghue, K.A. 2006. Genetic evaluation of female reproductive performance. http://www. bifconference.com/bif2002/Baker_Essay_ pdfs/Donoghue_02BIF.pdf> (15/04/2006).

Formigoni, I.B., Ferraz, J.B.S., Silva, J.A. II. V., Eler, J.P. e Brumatti, R.C. 2005. Valores econômicos para habilidade de permanência e probabilidade de prenhez aos 14 meses em bovinos de corte. Arq Bras Med Vet Zootecn, 57: 220-226.
Forni, S. and Albuquerque, L.G. 2005. Estimates of genetic correlations between days to calving and reproductive and weight traits in Nellore cattle. J Anim Sci, 83: 1511-1515.

Fries, L.A. 2003. Genética para um sistema de produção de ciclo curto. Em: Simpósio da Carne Bovina: da Produção ao Mercado Consumidor. Anais... UFRGS. São Borja, RS. 1: 1-17.

Garnero, A. del V., Lôbo, R.B., Bezerra, L.A.F., Bezerra, S.P.F., Corrado, M.P. e Schwengber, E.B. 1999. Estimativas de parâmetros genéticos de características reprodutivas na raça Nelore. Em: Reunião Anual da Sociedade Brasileira de Zootecnia, Anais... Sociedade Brasileira de Zootecnia. Porto Alegre, RS. 36: 157.

Ortiz Peña, C.D., Brito, F.V., Schenkel, F.S., Severo, J.L.P., Piccoli, M.L., Carvalheiro, R., Roso, V.M. e Cardoso, V. 2008. PAINT: Índice bioeconômico de fêmeas (RMat). Em: Congresso Brasileiro das Raças Zebuínas. Anais... Associação Brasileira dos Criadores de Zebu. Uberaba, MG. 7: 126-132.

Mercadante, M.E.Z., Lôbo, R.B., Borjas, A.L.R., Bezerra, L.A.F. e Oliveira, H.N. 1996. Estudo genético - quantitativo de características de reprodução e produção em fêmeas da raça Nelore. Em: Reunião Anual da Sociedade Brasileira de Zootecnia. Anais... Sociedade Brasileira de Zootecnia. Fortaleza, CE. 33: 155-157.

Notter, D.R. 1995. Maximizing fertility in animal breeding programs. Em: Congresso Brasileiro de Reprodução Animal, Anais... Colégio Brasileiro de Reprodução Animal. Belo Horizonte, MG. 11: 36.

Perotto, D. Habilidade materna em bovinos de corte. Em: Congresso Brasileiro das Raças Zebuínas. Anais... Associação Brasileira dos Criadores de Zebu. Uberaba, MG. 7: 81-101.

Ponzoni, R.W. and Newman, S. 1989. Developing breeding objectives for Australian Cashmere goats. Anim Prod, 49: 35-47.

Queiroz, S.A., Costa, G.Z., Oliveira, J.A. e Fries, L.A. 2009. Efeitos ambientais e genéticos sobre escores visuais e ganho de peso a desmama de animais formadores da raça Brangus. Rev Bras Zootecn, 38: 277-283.

Silva, J.A. II. V., Oliveira, H.N., Eler, J.P. e Ferraz, J.B.S. 2003. Análise genética da habilidade de permanência em fêmeas da raça Nelore. Rev Bras Zootecn, 32: 598-604. 


\section{TANAKA, NEVES, OLIVEIRA, CARVALHEIRO E QUEIROZ}

Silveira, J.C., Mcmanus, C., Mascioli, A.S., Silva, L.O.C., Silveira, A.C., Garcia, J.A.S. e Louvandini, H. 2004. Fatores ambientais e parâmetros genéticos para características produtivas e reprodutivas em um rebanho Nelore no estado do Mato Grosso do Sul. Rev Bras Zootecn, 33: 1432-1444

Teixeira, R.A., Albuquerque, L.G. e Fries, L.A.
2002. Comparações bioeconômicas entre três idades à primeira cobertura em novilhas Nelore. Ars Vet, 18: 197-203.

Valle, E.R., Andreotti, R. e Thiago, L.R.L.S. 1998. Estratégias para o aumento da eficiência reprodutiva e produtiva de bovinos de corte. Embrapa CNPGC. Documentos 71. Campo Grande. 80 pp.

Archivos de zootecnia vol. 61, núm. 236, p. 548. 